\title{
Is visual illusion decrement based on selective adaptation?
}

\author{
CLARE PORAC \\ University of Victoria, Victoria, British Columbia, Canada
}

\begin{abstract}
Illusion decrement is the reduction in the magnitude of visual geometric illusions with continued exposure, and it has been explained in two ways. The first explanation is the selective adaptation, or fatigue, of neural channels carrying orientation and/or spatial frequency information; the second explanation involves perceptual learning, in which the observer changes viewing strategy after continued exposure to a stimulus. Either mechanism could cause changes in the perception of a stimulus over time. One hundred twenty observers were tested in an illusiondecrement paradigm under exposure conditions that altered the amount of selective adaptation of specific neural channels. Observers were also measured on the magnitude of the transfer-ofdecrement effect. Both decrement and transfer of decrement occurred, but there was no significant difference across exposure conditions. In addition, the pattern of transfer differed from that observed in selective adaptation paradigms. These results argue against a neural adaptation interpretation of illusion decrement.
\end{abstract}

Illusion decrement is the reduction in measured illusion magnitude with continued exposure to a stimulus. It is demonstrated most often using the Müller-Lyer figure. Observers' measured illusion magnitudes decrease if they scan the stimulus for a short time. Five minutes of inspection of the standard Müller-Lyer figure routinely produces reductions in original illusion-magnitude scores of $15 \%-40 \%$ (see Coren \& Girgus, 1978a, for a review).

Illusion decrement is commonly explained as a form of perceptual learning in which observers reorganize their information-processing strategies while scanning an illusion figure (Coren \& Girgus, 1978a, 1978b); a reduction of the illusory effect is the result. This perceptual reorganization explanation is bolstered by the fact that reductions in illusion strength are greatest when observers actively explore the illusion figures with free eye movements (Festinger, White, \& Allyn, 1968). If some visual illusions, such as the Müller-Lyer figure, are based on eye-movement errors caused by the effect of converging lines (see Coren, 1986), then continued exposure with free scanning allows correction of these errors, and illusion magnitude decreases. A recalibration of informationprocessing strategies occurs on the basis of information from eye-movement corrections.

A competing explanation contends that decrement is the result of changes in the physiological structures responsible for the processing of form information, such as orientation-specific or spatial-frequency specific cortical cells. Some researchers have suggested that visual illu-

This research was funded by the Natural Sciences and Engineering Research Council of Canada. The assistance of Daniel Marshall in the collection of these data is gratefully acknowledged. Address reprint requests to C. Porac, Department of Psychology, University of Victoria, Victoria, British Columbia V8W 2Y2 Canada. sions containing lines of different orientations are caused by the interaction of orientation-tuned cortical neurons (Blakemore, Carpenter, \& Georgeson, 1970; Burns \& Pritchard, 1971). Continued exposure to a figure with contours oriented to elicit the maximal response from a group of cells causes a selective fatigue effect, or adaptation, of these cells (Howard, 1982). This adaptation weakens the effect of the neural interactions responsible for the illusion and results in the measured illusion-magnitude decrement.

There is strong evidence that Müller-Lyer decrement is a central effect, whether of structural (adaptation) or cognitive (information-processing) origin. It occurs in cyclopean viewing (Coren \& Porac, 1984) and demonstrates interocular transfer (Porac \& Coren, 1977). In addition, decrement reductions transfer between different MüllerLyer variants that share similar features (Coren \& Girgus, 1974; Porac \& Coren, 1985). This transfer-of-decrement finding supports the interpretation of a central learning component involved in illusion-magnitude reduction over time.

Recently, Long (1988) suggested that decrement and transfer-of-decrement paradigms are identical to selective adaptation paradigms that study the contribution of localized neural mechanisms to perceptual effects, such as ambiguous figure reversal. Both methodologies involve a stimulus inspection period of 2-5 min, followed by measurements on a test figure assumed to share certain features with the inspection figure. The selective adaptation approach interprets changes in the perception of the test figure as indicating the action of similar neural mechanisms in the test and inspection figures. On the other hand, results from transfer-of-decrement experiments have been interpreted from a cognitive perspective, which views the perceptual changes in the test figure as a short-term and 
long-term learning effect. Thus, operationally identical paradigms have not provided consistent evidence converging on one mechanism underlying time-related changes in various illusory percepts. Since existing experimental work supports both interpretations (Coren \& Girgus, 1974; Coren, Girgus, \& Schiano, 1986; Girgus, Coren, Durant, \& Porac, 1975; Long \& Toppino, 1981; Long, Toppino, \& Kostenbauder, 1983; Porac \& Coren, 1985; Toppino \& Long, 1987), Long (1988) expressed the need for additional research to clarify this interpretative problem with regard to specific illusion figures.

To gather further data about the mechanism underlying decrement in the Müller-Lyer illusion, I incorporated selective adaptation techniques, used primarily in the study of binocular and monocular neural networks, into an illusion-decrement paradigm. The orientation-specific cortical cells, said to contribute to intersecting-line visual distortions, are assumed to be driven both binocularly and monocularly (Blake, Overton, \& Lema-Stern, 1981; Blakemore \& Campbell, 1969). This combined methodology thus permits an exploration of the role of orientationspecific cortical cells, and their adaptation, as a mechanism in illusion decrement.

\section{METHOD}

\section{Subjects}

One hundred twenty undergraduate volunteers from the University of Victoria participated in the experiment. All observers had tested visual acuity in the normal range without correction and all were right-eyed sighting dominant as assessed by procedures devised by Porac and Coren (1981). The sighting-dominance testing was included to allow control of possible sighting-dominance effects in the decrement and transfer stages of the experiment (see Porac \& Coren, 1977).

\section{Stimuli and Procedures}

All observers viewed the standard Brentano version of the MüllerLyer illusion. The stimulus was black on a white background, with the overestimated portion made adjustable with a sliding apparatus. Observers judged the line length of the horizontal shaft by adjusting the overestimated section until both sides of the figure appeared to be of equal length. Measurements of illusion magnitude in millimeters were taken after each judgment from a ruler attached to the back of the figure. The horizontal shaft of the stationary portion of the Müller-Lyer figure was $80-\mathrm{mm}$ long. The figure had $30-\mathrm{mm}$ "wings" placed at an angle of $30^{\circ}$ to the horizontal shaft. The stimulus was presented at eye level at a viewing distance of $55 \mathrm{~cm}$; at this distance, the stationary portion of the stimulus subtended a visual angle of $8.28^{\circ}$. The background luminance of the stimulus display was $20.08 \mathrm{~cd} / \mathrm{m}^{2}$.

The experiment had two sections: a transfer and a decrement segment. First, each observer made a predecrement judgment of illusion magnitude, monocularly, with the right and then the left eye. Each subject underwent 3 min of decrement in which he or she scanned the Müller-Lyer figure with both sides set to physical equality. During scanning, the observers were instructed to move their eyes across the horizontal shaft from right to center to left and back again in a systematic fashion. Ilusion-magnitude judgments were made at 1-min intervals. Finally, each observer made two postdecrement judgments of illusion strength in the same manner as the predecrement judgment. The monocular order of the pre- and postdecrement judgments was counterbalanced across observers.

Observers were divided into five groups. Group 1 scanned binocularly. Groups 2 and 3 used monocular viewing, either with the right eye (Group 2) or with the left eye (Group 3). Groups 4 and 5 scanned the stimulus under right- and left-eye successive alternations. Observers in Group 4 scanned the stimulus for $1 \mathrm{~min}$ with the right eye, made a judgment, then scanned for 1 min with the left eye. The eye that started the decrement process was counterbalanced across observers. Observers in Group 5 scanned the stimulus for $30 \mathrm{sec}$ with the right eye, then switched for $30 \mathrm{sec}$ of scanning with the left eye before making a judgment of illusion magnitude. This second alternating group ensured that each eye received equal exposure during the decrement process. Once again, the eye that started the decrement process was counterbalanced across observers. The observers wore a pair of goggles, and monocular exposure during decrement (Groups 2-5) and pre- and postdecrement testing was accomplished by dropping a shutter across the goggle lens of the appropriate eye.

All observers made two predecrement and two postdecrement judgments (monocularly with the right and left eye) and four judgments during the decrement phase. Illusion-magnitude scores were computed by subtracting the observers' settings from the veridical extent of the stationary portion of the Müller-Lyer figure $(80 \mathrm{~mm})$.

\section{RESULTS}

\section{Illusion Magnitude and Decrement}

Studies of binocular vision using selective adaptation paradigms make specific predictions about the pooled neural activity and its perceptual consequences as a result of different exposure conditions (Blake et al., 1981; Wolfe, 1986; Wolfe \& Blake, 1985). Briefly, three classes of neurons are active: a right- and left-eye monocular pool and a binocular pool activated by input from either eye. This binocular pool is most active under binocular viewing, but since each eye has the capability of stimulating its portion of the pool, it is also active to a lesser extent under monocular exposure. Some models propose a fourth neural group, binocular "and" cells that are only active when both eyes are open (Wolfe, 1986).

One can combine these notions with the assumption that illusion magnitude is caused by the interaction of orientation-specific cells in the visual cortex, and that decrement results from their selective adaptation during prolonged exposure. Since these feature detectors can be driven both monocularly and binocularly, several predictions are possible. First, original illusion magnitude should be significantly greater under binocular than under monocular conditions. Second, the magnitude of illusion reduction should differ across the various exposure conditions. Decrement for the continuous binocular condition should exceed that of the two monocular conditions because more cells are responsive during binocular vision. The alternating binocular conditions, in which the right eye and the left eye are exposed in succession, should produce little or no decrement because neither the monocular nor the binocular pool undergoes selective prolonged exposure. These predictions are based on a sim- 
ple model of binocular interaction. However, they are consistent with the assumptions made by binocular network models and with the data on binocular summation, which indicate that binocular performance in a number of visual tasks is better than that expected from a simple summation of the two monocular responses (Blake \& Fox, 1973). Table 1 and Figure 1 show the resulting data relevant to these predictions.

Table 1 shows the mean original illusion magnitude (Minute 0 ) and the mean original percent illusion [Minute 0 $(\mathrm{mm}) / 80 \mathrm{~mm}]$ for each exposure group. An analysis of variance (ANOVA) indicated no significant group differences. The prediction that greater neural activity in the continuous binocular exposure condition would produce higher illusion scores was not supported. Previous work has shown that susceptibility to the Müller-Lyer distortion changes when experimental manipulations alter the involvement of neural mechanisms in the formation of the illusion. For example, altering the Müller-Lyer figure to, a dot form in order to reduce the contribution of neural mechanisms lowers original illusion scores by $65 \%$ of the expected magnitude (Girgus et al., 1975). However, in the present case, altering neural contribution by varying exposure conditions did not affect illusion scores significantly.

Figure 1 shows the change in illusion magnitude (in millimeters) over the decrement period. ANOVAs revealed that each group showed a significant change over time. The $F$ scores, all significant at $p<.01$, were binocular continuous $[F(3,69)=8.80]$, monocular right eye $[F(3,69)=6.87]$, monocular left eye $[F(3,69)=$ 6.16], alternating binocular, 60 sec/eye $[F(3,69)=6.38]$, and alternating binocular, 30 sec/eye $[F(3,69)=5.10]$. A combined ANOVA of the illusion-magnitude scores in the five exposure groups revealed no differences related to exposure group, nor any group $\times$ time interactions; however, there was, once again, a significant overall reduction in scores over time $[F(3,345)=30.66, p<.01]$. Thus, each group responded in an equivalent fashion during the decrement period. The lack of an exposure $x$ time interaction cannot be explained by a uniform shift in scale produced by different exposure conditions, since the original illusion-magnitude levels were similar across the five exposure conditions. Thus, the predecrement starting point was the same, irrespective of exposure condition.

Table 1

Mean Original Illusion-Magnitude Scores (Minute 0 and Percent Illusion) for Five Decrement Groups

\begin{tabular}{lcc} 
& \multicolumn{2}{c}{ Illusion } \\
\cline { 2 - 3 } Exposure & Magnitude* & Percent \\
\hline & Monocular & Decrement Group \\
Right Eye & 24.50 & 30.6 \\
Left Eye & 22.96 & 28.7 \\
& Binocular Decrement Group \\
Continuous & 26.13 & 32.7 \\
Alternate & & \\
60 sec/eye & 25.33 & 31.7 \\
30 sec/eye & 25.04 & 31.3 \\
\hline
\end{tabular}

*In millimeters

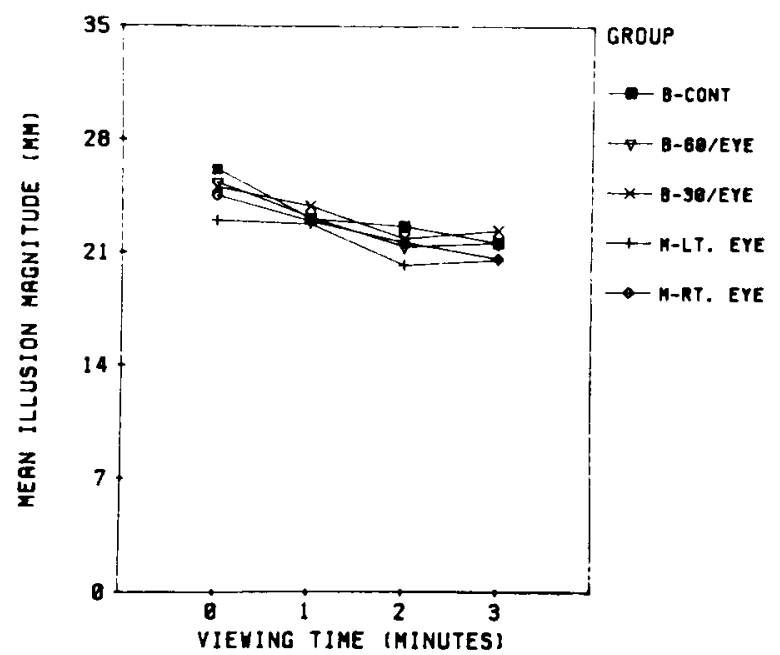

Figure 1. Mean illusion-magnitude scores for five decrement groups as a function of length of exposure. B-CONT: Binocular, continuous viewing; B-60/EYE: Binocular, alternating $60 \mathrm{sec} / \mathrm{eye}$; B-30 EYE: Binocular, alternating 30 sec/eye; M-LT. EYE: Monocular, left eye; M-RT. EYE: Monocular, right eye.

\section{Transfer of Decrement}

Each observer made judgments of illusion magnitude under right- then left-eye exposure before and after the decrement period. Significant reductions in illusion magnitude for both right- and left-eye exposure occurred in all groups from pretest to posttest $[F(1,115)=131.34$, $p<.01$ ]. There were no eye (right vs. left) or exposure group effects that significantly interacted with the pretest-posttest reduction. The average right-eye reduction was $4.95 \mathrm{~mm}$ and that for the left eye was $5.65 \mathrm{~mm}$.

Previous research on interocular transfer has indicated that a direct effect will differ from an indirect effect when the adaptation (decrement) is monocular (Blake et al., 1981). In other words, right-eye exposure leads to greater postadaptation changes in the right eye (direct effect) relative to the left eye (indirect effect). Blake et al. (1981) argued that during the adaptation period, the monocular neurons of the unexposed eye remain unadapted; therefore, when interocular transfer is measured, the magnitude of the indirect effect is based only on the adaptation of the shared binocular neural pool. In the exposed eye, both the monocular and the binocular neurons are adapted; hence, greater postadaptation changes are expected in the exposed eye. In the present case, the pretest-posttest difference in illusion magnitude (transfer of decrement) should be greater when measured in the eye exposed during decrement.

The first two rows of Table 2 show the transfer-ofdecrement results for the monocular exposure groups. Table 2 lists the transfer scores (pretest minus posttest) for both the right and the left eyes. Both the right- and the left-eye decrement condition produced significant preversus posttest changes $[F(1,23)=30.60, p<.01$, for the right-eye group; $F(1,23)=38.15, p<.01$, for the left-eye group]. There were no significant differences be- 
Table 2

Mean Transfer-of-Decrement (pretest minus posttest) for Five Decrement Groups

\begin{tabular}{lcc}
\hline & \multicolumn{2}{c}{ Mean Transfer* } \\
\cline { 2 - 2 } Exposure & Right Eye & Left Eye \\
\hline & Monocular & Decrement Group \\
Right Eye & 3.17 & 6.21 \\
Left Eye & 4.33 & 4.75 \\
& Binocular Decrement Group & \\
Continuous & 5.96 & 7.25 \\
Alternate & & \\
60 sec/eye & 4.92 & 4.88 \\
30 sec/eye & 6.37 & 5.17 \\
\hline
\end{tabular}

*In millimeters

tween the transfer scores (pretest minus posttest) for the left-eye decrement group; however, decrement on the right eye produced significantly greater reductions in illusion strength in the left eye $[t(23)=-2.38, p<.05]$ relative to that of the right eye. Left-eye decrement produced no difference between the direct (left-eye) and the indirect (right-eye) effects, whereas right-eye decrement resulted in transfer effects in the direction opposite to that predicted.

Blake et al. (1981) argued that the magnitude of the transfer-of-adaptation effect is based on the total number of neurons active during the adaptation process. Continuous binocular exposure generates activity in all neural units, binocular as well as monocular. Alternate eye exposure generates activity in monocular units but minimizes the involvement of binocular neural pools. In fact, binocular "and" cells would not undergo adaptation with alternate eye exposure. For these reasons, continuous binocular exposure during adaptation (decrement) should lead to greater changes, or larger aftereffects.

ANOVAs revealed that significant pre- to posttest reduction occurred in each of the three binocular groups $[F(1,23)=22.76, p<.01$ for continuous binocular decrement; $F(1,23)=25.62, p<.01$, for alternations of $60 \mathrm{sec} /$ eye; and $F(1,23)=26.95, p<.01$, for alternations of $30 \mathrm{sec} / \mathrm{eye}$. These data are shown in the lower half of Table 2. Additional analyses on the pretest-posttest difference scores indicated that the transfer effect was equivalent across the three groups. Again, predictions based on the adaptation of neural channels failed to account for the empirical results.

\section{DISCUSSION}

The present findings do not directly support a perceptual learning interpretation of illusion decrement, but they suggest that selective adaptation of cortical cells is not the sole explanation of decrement phenomena. If the magnitude of an adaptation effect rests on the activity of the numbers of neurons selectively adapted, differences in decrement magnitude as a function of visual exposure would have occurred. Equivalent illusion magnitude and decrement occurred in all exposure groups, even under alternating eye conditions with the least selective exposure of specific neural channels. These results tend to confirm those of Coren et al. (1986) and Girgus et al.(1975), who showed that experimental manipulations thought to alter the neural involvement in viewing the Müller-Lyer figure affect original illusion magnitude but not the shape of the decrement function. The present experiment produced identical original illusion magnitude and identical decrement reduction regardless of visual exposure. Presumptive variation in neural activity did not substantially affect Müller-Lyer decrement. In addition, monocular and/ or binocular exposure during decrement did not produce the same pattern of interocular transfer as found in selective adaptation paradigms. Major models of interocular transfer are based on results from threshold aftereffects, such as the contrast-sensitivity threshold (Blake et al., 1981). These models may not apply to suprathreshold aftereffects in which the selective exposure involves complex form-identification processes.

In the present experiment, a brief 3-min decrement exposure was used, unlike most previous research, in which 5- to 10-min exposures were used (Coren \& Girgus, 1974; Girgus et al., 1975; Porac \& Coren, 1977, 1985). Thus, the absolute duration of the adaptation phase was equivalent to the 2 to $3 \mathrm{~min}$ used in selective adaptation paradigms (Long et al., 1983; Toppino \& Long, 1987). Significant illusion reduction and transfer in this brief period indicates that the process underlying decrement occurred in the same time interval as the selective adaptation of cortical channels. However, this time factor is the only similarity found between the present results and those predicted by selective adaptation tenets.

\section{REFERENCES}

Blake, R. , Fox, R. (1973). The psychophysical inquiry into binocular summation. Perception \& Psychophysics, 14, 161-185.

BlaKe, R., OVERTON, R., \& Lema-STERn, S. (1981). Interocular transfer of visual aftereffects. Journal of Experimental Psychology: Human Perception \& Performance, 7, 367-381.

Blakemore, C., CAMPBell, F. W. (1969). On the existence of neurones in the human visual system selectively sensitive to the orientation and size of retinal images. Journal of Physiology, 203, 237-261.

Blakemore, C., Carpenter, R. H. S., \& Georgeson, M. A. (1970). Lateral inhibition between orientation detectors in the human visual system. Nature, 228, 37-39.

Burns, B. D., \& Pritchard, R. (1971). Geometrical illusions and the responses of neurones in the cat's visual cortex to angle patterns. Journal of Physiology, 213, 599-616.

Coren, S. (1986). An efferent component in the visual perception of direction and extent. Psychological Review, 93, 391-410.

CoRen, S., \& GrRGUS, J. S. (1974). Transfer of illusion decrement as a function of perceived similarity. Journal of Experimental Psychology, 102, 881-887.

COREN, S., \& GiRGUS, J. S. (1978a). Seeing is deceiving: The psychology of visual illusions. Hillsdale, NJ: Erlbaum.

COREN, S., \& Girgus, J. S. (1978b). Visual illusions. In R. Held, H. Leibowitz, \& H. L. Teuber (Eds.). Handbook of sensory physiology: Vol. 8. Perception (pp. 549-568). Berlin: Springer. 
Coren, S., Girgus, J. S., \& Schiano, D. (1986). Is adaptation of orientation-specific cortical cells a plausible explanation of illusion decrement? Bulletin of the Psychonomic Society, 24, 207-210.

Coren, S., \& Porac, C. (1984). Structural and cognitive components in the Müller-Lyer illusion assessed via Cyclopean presentation. Perception \& Psychophysics, 35, 313-318.

Festinger, L., White, C. W., \& Allyn, M. R. (1968). Eye movements and decrement in the Müller-Lyer illusion. Perception \& Psychophysics, 3, 376-382.

Girgus, J. S., Coren, S., Durant, M., \& Porac, C. (1975). The assessment of components involved in illusion formation using a longterm decrement procedure. Perception \& Psychophysics, 18, 144-148.

Howard, I. P. (1982). Human spatial orientation. New York: Wiley.

LoNG, G. M. (1988). Selective adaptation vs. transfer of decrement: The conjoint effects of neural fatigue and perceptual learning. Perception \& Psychophysics, 43, 207-209.

LoNG, G.M., \& TopPINo, T. C. (1981). Multiple representations of the same reversible figure: Implications for cognitive decisional interpretations. Perception, 10, 231-234.

Long, G. M., Toppino, T. C., \& Kostenbauder, J. F. (1983). As the cube turns: Evidence for two processes in the perception of a dynamic reversible figure. Perception \& Psychophysics, 34, 29-38.
Porac, C., Coren, S. (1977). The assessment of motor control in sighting dominance using an illusion decrement procedure. Perception \& Psychophysics, 21, 341-346.

Porac, C., COREN, S. (1981). Lateral preferences and human behavior. New York: Springer.

Porac, C., Coren, S. (1985). Transfer of illusion decrement: The effects of global versus local figural variations. Perception \& Psychophysics, 37, 515-522.

Toppino, T. C., LONG, G. M. (1987). Selective adaptation with reversible figures: Don't change that channel. Perception \& Psychophysics, 42, 37-48.

WolfE, J. M. (1986). Stereopsis and binocular rivalry. Psychological Review, 93, 269-282.

WolfE, J. M., BlAKe, R. (1985). Dissecting the binocular visual system with psychophysical tools. In D. Rose \& V. Dobson (Eds.), Models of the visual cortex (pp. 192-199). Chichester, England: Wiley.

(Manuscript received July 6, 1988; revision accepted for publication January 25,1989 .)

\title{
Announcement
}

\author{
30th Annual Meeting of the Psychonomic Society \\ Atlanta, Georgia \\ November 17-19, 1989
}

The 30th Annual Meeting of the Psychonomic Society will be held in Atlanta, Georgia, November 17. 19,1989 . The meetings will begin Friday morning and continue until Sunday at noon. The headquarters hotel will be the Hyatt Regency.

The program and hotel reservation cards will be mailed to members and associates in September. A copy of the program will be published in the November issue of the Bulletin of the Psychonomic Society.

For further information, please contact the secretary-treasurer of the Society: Michael E. Rashotte, Department of Psychology, Florida State University, Tallahassee, FL 32306-1051 (Telephone: 904-644-2040; BITNET: Rashot@FSU). 